\title{
Circular degree choosability
}

\author{
Serguei Norine ${ }^{*} \quad$ Xuding Zhu ${ }^{\dagger}$
}

Submitted: Dec 19, 2007; Accepted: Jul 27, 2008; Published: Aug 4, 2008

Mathematics Subject Classification: 05C15

\begin{abstract}
We extend a characterization of degree-choosable graphs due to Borodin [1], and Erdős, Rubin and Taylor [2], to circular list-colorings.
\end{abstract}

\section{Introduction}

Let $G=(V(G), E(G))$ be a graph. A list assignment $L$ for $G$ is a mapping which assigns to each vertex $v$ of $G$ a set of non-negative integers $L(v)$. An $L$-coloring of $G$ is a proper coloring $c$ of $G$ such that $c(v) \in L(v)$ for every $v \in V(G)$. A graph $G$ is degree-choosable if $G$ admits an $L$-coloring for every list assignment $L$, such that $|L(v)| \geq \operatorname{deg}(v)$ for all $v \in V(G)$. Borodin [1] and Erdős, Rubin and Taylor [2] characterized degree-choosable graphs as follows.

Theorem 1. A graph $G$ is not degree-choosable if and only if each of the blocks of $G$ is a clique or an odd cycle (i. e. $G$ is a Gallai tree).

In this paper we prove an analogue of Theorem 1 for circular colorings. Informally, a circular coloring is a coloring of the vertices of the graph by points of a (possibly discrete) circle, such that the circular distance between the colors assigned to adjacent vertices of the graph is bounded from below. Circular colorings have attracted considerable attention over the last decade (see [9] for a survey of the subject). Circular version of list-colorings has been recently introduced by Mohar [4] and Zhu [8], and has been since studied in [3, $5,6,7]$, among others.

Let us now formally present the relevant definitions and notation. Let $p$ be a positive integer. For an integer $a$ we denote by $[a]_{p}$ the remainder of $a$ modulo $p$. Define $S_{p}$ as $\{0,1, \ldots, p-1\}$. For $a, b \in S_{p}$, the interval $[a, b]_{p}$ is defined as

$$
[a, b]_{p}=\{a, a+1, a+2, \cdots, b\}
$$

*Department of Mathematics, Princeton University, Princeton, NJ 08540-1000. Partially supported by NSF grants DMS-0200595 and DMS-0701033.

${ }^{\dagger}$ Department of Applied Mathematics, National Sun Yat-sen University, Kaohsiung, Taiwan 80424, and National Center for Theoretical Sciences. Partially supported by National Science Council of R.O.C. through grant NSC95-2115-M-110-013-MY3 
where the additions are modulo $p$. The circular distance $|a-b|_{p}$ between $a$ and $b$ is defined as

$$
|a-b|_{p}=\min \left\{[a-b]_{p},[b-a]_{p}\right\}=\min \left\{\left|[a, b]_{p}\right|,\left|[b, a]_{p}\right|\right\}-1 .
$$

Let $q$ be a positive integer, such that $p \geq 2 q$, and let $G=(V(G), E(G))$ be a graph. A $(p, q)$-coloring of $G$ is a function $c: V(G) \rightarrow S_{p}$, such that for every edge $u v \in E(G)$ we have $|c(u)-c(v)|_{p} \geq q$. Given a list assignment $L$, an $L-(p, q)$-coloring of $G$ is a $(p, q)$-coloring that is also an $L$-coloring. We say $G$ is $L$ - $(p, q)$-colorable if there is an $L$ - $(p, q)$-coloring of $G$. Let $l: V(G) \rightarrow\{0,1, \ldots, p\}$ be a function. We say that a listassignment $L$ for $G$ is an l-p-list assignment if $L(v) \subseteq S_{p}$ and $|L(v)| \geq l(v)$ for every $v \in V(G)$. We say that $G$ is $l$ - $(p, q)$-choosable if $G$ is $L$ - $(p, q)$-colorable for every l-p-list assignment $L$ for $G$.

We say that a graph $G$ is circular $q$-degree-choosable if $G$ is $l_{q^{-}}(p, q)$-choosable for every integer $p \geq(\Delta(G)-1)(2 q-1)+1$, where for $v \in V(G)$

$$
l_{q}(v)=(\operatorname{deg}(v)-1)(2 q-1)+1 .
$$

Observe that $l_{1}(v)=\operatorname{deg}(v)$. So a graph is circular 1-degree-choosable if and only if it is degree-choosable. In the following, we only consider circular $q$-degree-choosability for $q \geq 2$. We will show that for any integers $q_{1}, q_{2} \geq 2$, a graph $G$ is circular $q_{1}$-degreechoosable if and only if $G$ is circular $q_{2}$-degree-choosable. We say that a graph is circular degree-choosable if it is circular $q$-degree-choosable for some (and hence for all) integer $q \geq 2$.

Given a graph $G$, the heart of $G$ is the graph obtained from $G$ by repeatedly deleting degree one vertices. In this paper we characterize circular degree-choosable graphs as follows.

Theorem 2. A connected graph $G$ is not circular degree-choosable if and only if $G$ is a tree, or the heart of $G$ is an odd cycle, or $G$ is isomorphic to $K_{4}$.

In [3] Havet et al. considered notions of circular degree-choosability that are similar to, but distinct from ours. They defined a graph $G$ to be circularly $(2 d-2)$-choosable if $G$ is $l_{q}^{\prime}-(p, q)$-choosable for all integers $q$ and $p \geq(2 \Delta(G)-2) q$, where $l_{q}^{\prime}(v)=\max \{(2 \operatorname{deg}(v)-$ 2) $q, 1\}$ for $v \in V(G)$. They conjectured a characterization of circularly $(2 d-2)$-choosable graphs, namely, that a graph is circularly $(2 d-2)$-choosable unless it is a tree or an odd cycle. Note that such a characterization is not implied by Theorem 2, nor would it imply Theorem 2. Our notion of circular degree-choosability is more restrictive, but the class of graphs that are conjectured to be circularly $(2 d-2)$-choosable is larger. Our definition of circular degree choosability appears to us to be more natural. In particular, circular $q$-degree-choosability inherits the following property from degree choosability: For a given integer $q$ the function $l_{q}$, defined in (1), is the minimum function of $\operatorname{deg}(v)$, such that $G$ is $l_{q^{-}}(p, q)$-choosable for all $p \geq \max _{v \in V(G)} l_{q}(v)$, if, and only if, some induced subgraph $H$ of $G$ is $l_{q^{-}}(p, q)$-choosable.

The remainder of the paper is devoted to the proof of Theorem 2. In Section 2 we introduce results from $[2,5,7]$ that we will utilize in our proof and prove a couple of simple auxiliary lemmas. The proof itself is presented in Section 3. 


\section{Prerequisites}

Our proof of Theorem 2 follows the general outline of the proof of characterization of degree-choosable graphs in [2]. We prove that a graph $G$ is circular $q$-degree-choosable if $G$ has an induced subgraph $H$ which is circular $q$-degree-choosable. Then we show that if the heart of $G$ is non-empty and distinct from an odd cycle then $G$ contains an induced subgraph with a simple, particular structure. Finally, we prove that graphs with such a structure are circular $q$-degree-choosable. In this section we complete the first two steps of the proof, and introduce the results that we will utilize in the last step. Let us start by introducing some additional notation. For a positive integer $p$ and $x \in S_{p}$ denote by $B_{p, q}(x)$ the set of elements of $S_{p}$ at circular distance less than $q$ from $x$, i.e., $B_{p, q}(x)=[x-q+1, x+q-1]_{p}$. We write $B(x)$ instead of $B_{p, q}(x)$, when the values of $p$ and $q$ are understood from the context.

Lemma 3. Let $q \geq 1$ be an integer, let $G$ be a graph and let $H$ be an induced subgraph of $G$. If $H$ is circular $q$-degree-choosable, then so is $G$.

Proof. It suffices to prove the lemma in the case when $H$ is obtained from $G$ by deleting some vertex $w \in V(G)$. Let $p \geq(\Delta(G)-1)(2 q-1)+1$ be an integer. Let $L$ be an $l_{q}$-p-list assignment for $G$, where $l_{q}$ is defined as in (1). We will prove that $G$ admits an $L$ - $(p, q)$-coloring. Let $c_{0} \in L(w)$ be chosen arbitrarily. Define a list assignment $L^{\prime}$ for $H$ as follows: Let $L^{\prime}(v)=L(v) \backslash B_{p, q}\left(c_{0}\right)$ for every $v \in V(H)$, such that $v w \in E(G)$, and let $L^{\prime}(v)=L(v)$, otherwise. Then $\left|L^{\prime}(v)\right| \geq\left(\operatorname{deg}_{H}(v)-1\right)(2 q-1)+1$ for every $v \in V(H)$, and thus there exists an $L^{\prime}-(p, q)$-coloring $c$ of $H$. We extend $c$ to a coloring of $G$ by setting $c(w)=c_{0}$. Then $c$ is an $L-(p, q)$-coloring by the choice of $L^{\prime}$.

In proving the "only if" direction of Theorem 2 we will use the following partial converse of Lemma 3.

Lemma 4. Let $q \geq 1$ be an integer, let $G$ be a graph, and let $H$ be obtained from $G$ by deleting a vertex $u \in V(G)$, such that $u$ has a unique neighbor $w \in V(G)$. Let $l: V(H) \rightarrow \mathbb{Z}_{+}$be such that $H$ is not $l$ - $(p, q)$-choosable for all sufficiently large integers $p$. Let $l^{\prime}$ be obtained from $l$ by setting $l^{\prime}(u)=1, l^{\prime}(w)=l(w)+2 q-1$, and $l^{\prime}(v)=l(v)$ for all $v \in V(G) \backslash\{u, w\}$. Then $G$ is not $l^{\prime}-(p, q)$-choosable for all sufficiently large integers $p$.

Proof. Let $p_{0} \geq(2 q-1) l(w)+1$ be chosen so that for every integer $p \geq p_{0}$ the graph $H$ is not $l-(p, q)$-choosable. Consider arbitrary $p \geq p_{0}$. By the choice of $p$, there exists an $l$-p-list assignment $L$ for $H$, such that $H$ is not $L-(p, q)$-colorable. By the choice of $p_{0}$ there exists $c \in S_{p} \backslash\left(\bigcup_{a \in L(w)} B_{p, q}(a)\right)$. We have $B_{p, q}(c) \cap L(w)=\emptyset$. Let $L^{\prime}(u)=\{c\}$, $L^{\prime}(w)=L(w) \cup B_{p, q}(c)$, and let $L^{\prime}(v)=L(v)$ for $v \in V(G) \backslash\{u, w\}$. Then $L^{\prime}$ is an $l^{\prime}$-p-list assignment for $G$, and, clearly, there exists no $L^{\prime}-(p, q)$-coloring of $G$.

In [2], typical induced subgraphs of graphs that contain a block distinct from a clique or an odd cycle are described as follows. 
Lemma 5. [2] Let $G$ be a graph that contains a block distinct from a clique or an odd cycle. Then $G$ contains an induced subgraph $H$, such that $H$ is an even cycle with at most one chord.

The next description of typical induced subgraphs of graphs with the heart non-empty and distinct from an odd cycle follows immediately from Lemma 5.

Corollary 6. Let $G$ be a connected graph such that $G$ is not a tree and the heart of $G$ is not an odd cycle. Then $G$ contains an induced subgraph $H$, such that

- $H$ is a clique on 4 vertices, or

- $H$ is an even cycle with at most one chord, or

- $H$ consists of two odd cycles $C_{1}$ and $C_{2}$ joined by a path $P$, such that $P$ is internally disjoint from $C_{1}$ and $C_{2}$, and $C_{1}$ and $C_{2}$ are vertex disjoint, unless $P$ has zero length, in which case $C_{1}$ and $C_{2}$ share a single vertex.

Proof. If the heart of $G$ contains a block that is not an odd cycle, then the lemma follows from Lemma 5. If not, then $G$ contains two blocks, each of which is an odd cycle. By joining these blocks by an induced path in $G$ (possibly of length zero), we obtain the required subgraph $H$.

By Lemma 3 and Corollary 6, it suffices to prove Theorem 2 for graphs with simple structure. We will use the following results on circular colorings of trees and cycles in further analysis of colorings of these graphs.

Lemma 7. [7] Let $T$ be a tree, let $p \geq 2 q$ be positive integers and let $l: V(T) \rightarrow$ $\{0,1, \ldots, p\}$. Then $T$ is $l-(p, q)$-choosable if and only if for each subtree $T^{\prime}$ of $T$ we have

$$
\sum_{v \in V\left(T^{\prime}\right)} l(v) \geq 2 q\left(\left|V\left(T^{\prime}\right)\right|-1\right)+1
$$

Lemma 8. [5] Let $p \geq 2 q$ be positive integers. Let $G$ be an even cycle, and let $l(v)=2 q$ for every $v \in V(G)$. Then $G$ is $l$ - $(p, q)$-choosable.

\section{Proof of Theorem 2}

In this section we prove Theorem 2. We prove the "if" direction of the theorem by considering graphs that serve as outcomes of Corollary 6. We start by disposing of the first outcome.

Lemma 9. Suppose $q \geq 2, p \geq 4 q$ and $L$ is a list assignment of $K_{4}$ with $|L(x)| \geq 4 q-1$ for each vertex $x$. Then $G$ has an $L-(p, q)$-coloring. 
Proof. Without loss of generality, we may assume that $0 \in L\left(v_{1}\right)$ and $1 \notin L\left(v_{2}\right)$. Color $v_{1}$ with 0 . Let $c_{1}$ be the least color in $\cup_{i=2}^{4} L\left(v_{i}\right) \backslash[0, q-1]_{p}$. If $c_{1} \in L\left(v_{i}\right)$ for some $i \neq 2$, then color $v_{i}$ (say $v_{3}$ ) with color $c_{1}$. Otherwise, color $v_{2}$ with color $c_{1}$. At this moment, there are two uncolored vertices, that are either $v_{2}, v_{4}$ or $v_{3}, v_{4}$.

In the former case, $c_{1} \notin L\left(v_{3}\right) \cup L\left(v_{4}\right)$. Hence for $i=3,4, L\left(v_{i}\right) \backslash\left[0, c_{1}+q-1\right]_{p}$ contains at least $2 q$ colors. Let $c_{2}$ be the least color in $\left(L\left(v_{3}\right) \cup L\left(v_{4}\right)\right) \backslash\left[0, c_{1}+q-1\right]_{p}$. Assume $c_{2} \in L\left(v_{3}\right)$, then color $v_{3}$ with color $c_{2}$. Then $L\left(v_{4}\right) \backslash\left[0, c_{2}+q-1\right]_{p}$ contains at least $q$ colors. Color $v_{4}$ with the least color in $L\left(v_{4}\right) \backslash\left[0, c_{2}+q-1\right]_{p}$, we obtain an $L$ - $(p, q)$-coloring of $K_{4}$.

In the later case, let $c_{2}$ be the least color in $\left(L\left(v_{2}\right) \cup L\left(v_{4}\right)\right) \backslash\left[0, c_{1}+q-1\right]_{p}$. If $c_{2} \in L\left(v_{4}\right)$, then color $v_{4}$ with color $c_{2}$. Since $1 \notin L\left(v_{2}\right)$, we conclude that $L\left(v_{2}\right) \backslash\left[0, c_{2}+q-1\right]_{p}$ contains at least $q$ colors. Color $v_{2}$ with the least color in $L\left(v_{2}\right) \backslash\left[0, c_{2}+q-1\right]_{p}$, we obtain an $L$ - $(p, q)$-coloring of $K_{4}$. If $c_{2} \notin L\left(v_{4}\right)$, then color $v_{2}$ with color $c_{2}$. Now $L\left(v_{4}\right) \backslash\left[0, c_{2}+q-1\right]_{p}$ contains at least $q$ colors. Color $v_{4}$ with the least color in $L\left(v_{4}\right) \backslash\left[0, c_{2}+q-1\right]_{p}$, we obtain an $L-(p, q)$-coloring of $K_{4}$.

Corollary 10. Let $G$ be a connected graph with $V(G) \geq 5$ and suppose that $G$ contains a subgraph isomorphic to $K_{4}$. Then $G$ is circular $q$-degree choosable for every integer $q \geq 2$.

Proof. We need to prove that $G$ is $l_{q^{-}}(p, q)$-choosable for all integers $p \geq(\Delta(G)-1)(2 q-$ $1)+1$, and the function $l_{q}$ as in (1). By repeating the argument in the proof of Lemma 3 , we can see that it suffices to prove that some subgraph $H$ is $l_{q}^{H}-(p, q)$-choosable, where the superscript $H$ indicates that the degrees in the formula (1) for $l_{q}$ are taken in $H$. Choose $H$ isomorphic to $K_{4}$. Note that $\Delta(G) \geq 4$, and, thus, $p \geq 4 q$. The corollary now follows from Lemma 9.

When considering two of the remaining outcomes of Corollary 6, we produce the required coloring by precoloring some vertices and applying a variant of Lemma 7 to color the remaining ones. Unfortunately, for our purposes Lemma 7 is not always sufficient. Our next goal is to prove a slightly more technical result.

Lemma 11. Let $T$ be a tree, and let $p$ and $q$ be positive integers, such that $p \geq 4 q-1$. Then $T$ is $L$ - $(p, q)$-choosable for every list assignment $L$ with the following properties

(a) $L(v) \subseteq S_{p}$ for every $v \in V(T)$,

(b) $\sum_{v \in V\left(T^{\prime}\right)}|L(v)| \geq 2 q\left(\left|V\left(T^{\prime}\right)\right|-1\right)+1$ for each proper subtree $T^{\prime}$ of $T$,

(c) $\sum_{v \in V(T)}|L(v)| \geq 2 q(|V(T)|-1)$, and if the equality holds, then $L(t)$ is not an interval for some leaft of $T$.

Proof. If the inequality in the condition (c) does not hold with equality then the lemma follows from Lemma 7. Thus, we assume that it does hold with equality, and that $L(t)$ is not an interval for some leaf $t$ of $T$. Let $u$ be the unique neighbor of $t$ in $T$. Define a list assignment $L^{\prime}$ for $T-t$ as follows: Let $L^{\prime}(u)=\left\{x \in L(u) \mid L(t) \nsubseteq B_{p, q}(x)\right\}$, and let $L^{\prime}(v)=L(v)$ for all $v \in V(T) \backslash\{w, u\}$. Note that $L^{\prime}$ satisfies conditions of Lemma 7 , 
as long as $\left|L^{\prime}(u)\right| \geq|L(u)|+|L(t)|-2 q+1$. Moreover, any $L^{\prime}$ - $(p, q)$-coloring of $T-w$ extends to an $L$ - $(p, q)$-coloring of $T$ by the choice of $L^{\prime}$.

Thus, it remains to show that $\left|L^{\prime}(u)\right| \geq|L(u)|+|L(t)|-2 q+1$. By (b) and our assumptions, we have $|L(t)| \leq 2 q-1$. Therefore, we assume that there exists $z \in$ $L(u) \backslash L^{\prime}(u)$. Then $L(t) \subseteq B(z)$. Let $x$ and $y$ be the elements of $L(t)$ closest to $z-q+1$ and $z+q-1$, respectively. We have $L(t) \subsetneq[x, y]_{p}$ and therefore $|y-x|_{p} \geq|L(t)|$. Finally, we have $L(u) \backslash L^{\prime}(u)=\bigcap_{s \in L(t)} B(s) \subseteq B(x) \cap B(y)$, and $|B(x) \cap B(y)|=2 q-1-|y-x|_{p} \leq$ $2 q-1-|L(t)|$. The desired inequality follows.

Next we prove another technical result. It will later allow us to precolor vertices of the graph in such a way that sufficiently many colors remain available for the remaining vertices, and Lemma 11 is applicable.

Lemma 12. Let $p$ and $q$ be positive integers, such that $p \geq 4 q-1$. Let $w: S_{p} \rightarrow\{0,1,2\}$ be a weight function, such that $w\left(S_{p}\right)=4 q$, where $w\left(S_{p}\right)=\sum_{i \in S_{p}} w(i)$. Let

$$
A=\left\{a \in S_{p} \mid w\left(B_{p, q}(a)\right) \geq 2 q\right\} .
$$

Then $|A| \leq 3 q-1$. Moreover, if $|A|=3 q-1$ then $w(x) \in\{0,2\}$ for every $x \in S_{p}$, and either $q=1$, or there exists $a \in A$ such that $\operatorname{supp}(w) \backslash B_{p, q}(a)$ is not an interval, where $\operatorname{supp}(w)=\{i \mid w(i) \neq 0\}$.

Proof. We prove the lemma for fixed $q$ by induction on $p$. We start by considering the base case $p=4 q-1$. Let $X=\left\{(a, b)|a \in A| a-,\left.b\right|_{p}=q\right\}$. Then $|X|=2|A|$. On the other hand, $\left|\left\{a \in S_{p} \mid\left(a, b_{0}\right) \in X\right\}\right| \leq 2$ for every $b_{0} \in S_{p}$. The inequality is strict for every $b_{0} \in \operatorname{supp}(w)$, as $B\left(b_{0}-q\right), B\left(b_{0}+q\right)$ and $\left\{b_{0}\right\}$ are pairwise disjoint subsets of $S_{p}$. Thus

$$
2|A| \leq|X| \leq 2(4 q-1)-|\operatorname{supp}(w)|
$$

It follows that $|A| \leq 3 q-1$.

Suppose now that $|A|=3 q-1$. Then $|\operatorname{supp}(w)|=2 q$, and therefore $w(x) \in\{0,2\}$ for every $x \in S_{p}$. If $\operatorname{supp}(w)$ is an interval, then $A=\operatorname{supp}(w)$, so $|A|=2 q$ and, thus, $q=1$.

Assume $\operatorname{supp}(w)$ is not an interval. Then there exists $x_{0} \in S_{p}$, such that $\operatorname{supp}(w) \backslash$ $B\left(x_{0}\right)$ is not an interval. If $x_{0} \in A$, or $q=1$, then the lemma holds, and so we assume $x_{0} \notin A$ and $q>1$.

Since $|X|=2(4 q-1)-|\operatorname{supp}(w)|$, then, using the argument from the first paragraph of the proof, we derive that for $a \in \operatorname{supp}(w)$, exactly one of $a-q$ and $a+q$ is in $A$, and for $a \notin \operatorname{supp}(w)$, we have $a-q, a+q \in A$. To simplify the notation we assume for the rest of the paragraph that $x_{0}=0$. Since $0 \notin A$, we conclude that $q, 3 q-1 \in \operatorname{supp}(w)$ and $2 q-1,2 q \in A$. Note that $\operatorname{supp}(w) \backslash B(2 q)$ and $\operatorname{supp}(w) \backslash B(2 q-1)$ can not be contained in a common interval of $\operatorname{supp}(w)$, as such an interval would have to contain $q$ and $3 q-1$ and would have to have length at least $2 q$, in contradiction with the assumption that $\operatorname{supp}(w)$ is not an interval (as $q$ is bigger than 1). Thus either one of $\operatorname{supp}(w) \backslash B(2 q)$ and $\operatorname{supp}(w) \backslash B(2 q-1)$ is not an interval, in which case the lemma holds, or $\operatorname{supp}(w) \backslash B(2 q)=$ $\{q\}$ and $\operatorname{supp}(w) \backslash B(2 q-1)=\{3 q-1\}$. In the second case, we have $w(q-1)=0$, and, 
thus $4 q-2 \in A$. It remains to note that $B(4 q-2) \cap B(2 q-1)=\emptyset$, and, therefore, $2 q \leq w(B(4 q-2))=w(2 q-1) \leq 2$. This contradicts our assumption that $q>1$, and so the proof in the base case is finished.

It remains to consider the induction step. If $\operatorname{supp}(w)=S_{p}$ then $p=4 q, w(x)=1$ for every $x \in S_{p}$, and the lemma trivially holds as $|A|=\emptyset$. Thus we assume $S_{p} \backslash \operatorname{supp}(w) \neq \emptyset$. We construct an auxiliary graph $H$ with $V(H)=S_{p}$, joining $x, y \in S_{p}$ by an edge in $H$ if, and only if, $|x-y|_{p} \geq 2 q-1$. The graph $H$ is connected, and so there exist $x \in \operatorname{supp}(w), y \notin \operatorname{supp}(w)$ such that $x y \in E(H)$. We have $w(B(y-q+1) \cap B(y+q-1))=0$, and $w(B(y-q+1) \cup B(y+q-1)) \leq 4 q-w(x)$. Therefore, not both of $y-q+1$ and $y+q-1$ belong to $A$. We assume, by symmetry, that $y=p$ and that $p-q+1 \notin A$. Consider the restriction $w^{\prime}$ of $w$ to $S_{p-1}$, and let $A^{\prime}=\left\{a \in S_{p-1} \mid w\left(B_{p-1, q}(a)\right) \geq 2 q\right\}$. Let $\phi: S_{p} \rightarrow S_{p-1}$ be defined as $\phi(z)=z$ if $z \leq p-q$, and $\phi(z)=z-1$ if $z \geq p-q+1$. Then $\phi(A) \subseteq A^{\prime}$, as $B_{p, q}(z) \cap S_{p-1} \subseteq B_{p-1, q}(\phi(z))$ for every $z \in S_{p}$. Moreover, we have $p-q+1 \notin A$, and, thus, $\phi$ is injective when restricted to $A$. It follows that $|A| \leq\left|A^{\prime}\right|$, and $A^{\prime} \leq 3 q-1$, by the induction hypothesis. The remaining conclusions of the lemma also follow straightforwardly from the induction hypothesis applied to $w^{\prime}$ and $A^{\prime}$.

Finally, we are ready to prove Theorem 2.

Proof of Theorem 2. We start by proving the "if" direction of the theorem. If $G$ contains a subgraph isomorphic to $K_{4}$ then the result follows from Corollary 10. Therefore, by Lemma 3 and Corollary 6 , it suffices to prove that $G$ is $q$-degree-choosable if $G$ is isomorphic to one of the other outcomes of Corollary 6 . Let $p \geq(\Delta(G)-1)(2 q-1)+1$ be a positive integer, and let $L$ be an $l_{q}$-p-list assignment for $G$, where $l_{q}$ is defined as in (1). It suffices to prove that $G$ is $L$ - $(p, q)$-colorable for all such $p$ and $L$. If $G$ is an even cycle then the result follows from Lemma 8.

Suppose next that $G$ is an even cycle with a single chord $u u^{\prime}$. Let $s$ and $t$ be the neighbors of $u$ in the cycle. For $x \in S_{p}$ let $w(x)=\chi_{L(s)}(x)+\chi_{L(t)}(x)$. (Here $\chi_{X}: S_{p} \rightarrow$ $\{0,1\}$ denotes the characteristic function of a subset $X$ of $S_{p}$.) By Lemma 12, applied to $w$, we conclude that there exists $c_{0} \in L(u)$ such that $\left|B\left(c_{0}\right) \cap L(s)\right|+\left|B\left(c_{0}\right) \cap L(t)\right| \leq 2 q-1$. Let $L^{\prime}$ be a list assignment for $G \backslash u$, such that $L^{\prime}(v)=L(v) \backslash B\left(c_{0}\right)$ for $v \in\left\{u^{\prime}, s, t\right\}$, and let $L^{\prime}(v)=L(v)$, otherwise. Then $\left|L^{\prime}\left(u^{\prime}\right)\right| \geq\left|L\left(u^{\prime}\right)\right|-2 q+1=2 q$, and $\left|L^{\prime}(s)\right|+\left|L^{\prime}(t)\right| \geq$ $|L(s)|+|L(t)|-2 q+1=2 q+1$. It follows that $L^{\prime}$ satisfies the conditions of Lemma 7 , and, thus, there exists an $L^{\prime}$ - $(p, q)$-coloring of $G \backslash u$, which can be extended to an $L$ - $(p, q)$ coloring of $G$ by coloring $u$ in color $c_{0}$.

It remains to prove the claim in the case when $G$ consists of two odd cycles $C_{1}$ and $C_{2}$ joined by a path $P$, such that $C_{1}, C_{2}$ and $P$ satisfy the conditions in the statement of Corollary 6 . Let $u_{1}$ and $u_{2}$ be the vertices that $P$ shares with $C_{1}$ and $C_{2}$, respectively. Let $s_{i}$ and $t_{i}$ be the neighbors of $u_{i}$ in $C_{i}$ for $i \in\{1,2\}$. Let $R_{i}$ be the set of colors $c$ in $L\left(u_{i}\right)$, such that either $\left|B(c) \cap L\left(s_{i}\right)\right|+\left|B(c) \cap L\left(t_{i}\right)\right|>2 q$; or $\left|B(c) \cap L\left(s_{i}\right)\right|+\left|B(c) \cap L\left(t_{i}\right)\right|=2 q$, and both $\left|B(c) \cap L\left(s_{i}\right)\right|$ and $\left|B(c) \cap L\left(t_{i}\right)\right|$ are intervals in $S_{p}$. Note that if $c \in L\left(u_{i}\right) \backslash R_{i}$ then every $L$ - $(p, q)$-coloring of $G \backslash\left(C_{i} \backslash u_{i}\right)$, where $u_{i}$ is colored in color $c$, can be extended to an $L-(p, q)$-coloring of $G$ by Lemma 11. For $x \in S_{p}$ let $w_{i}(x)=\chi_{L\left(s_{i}\right)}(x)+\chi_{L\left(t_{i}\right)}(x)$. By Lemma 12 applied to $w_{i}$, we conclude that $\left|R_{i}\right| \leq 3 q-2$. 
We are now ready to construct an $L-(p, q)$-coloring of $G$. Let $L^{\prime}$ be the following list assignment for $P$. Let $L^{\prime}\left(u_{1}\right)=L\left(u_{1}\right) \backslash R_{1}, L^{\prime}\left(u_{2}\right)=L\left(u_{2}\right) \backslash R_{2}$. If $u_{1}=u_{2}$ then let $L^{\prime}\left(u_{1}\right)=L\left(u_{1}\right) \backslash R_{1} \backslash R_{2}$, instead. Let $L^{\prime}(v)=L(v)$ for $v \in V(P) \backslash\left\{u_{1}, u_{2}\right\}$. We have $\left|L^{\prime}\left(u_{1}\right)\right|+\left|L^{\prime}\left(u_{2}\right)\right| \geq 2((4 q-1)-(3 q-2))=2 q+2$ if $u_{1} \neq u_{2}$, and we have $\left|L^{\prime}\left(u_{1}\right)\right| \geq 6 q-2-2(3 q-2)=2$ if $u_{1}=u_{2}$. Thus, $L^{\prime}$ satisfies the requirements of Lemma 7 , and so there exists an $L^{\prime}-(p, q)$-coloring of $P$. As noted above, such an $L^{\prime}$ $(p, q)$-coloring can be extended to an $L$ - $(p, q)$-coloring of $G$.

It remains to prove that if a graph $G$ is a tree, or $G$ has an odd cycle as its heart, or $G$ is isomorphic to $K_{4}$, then for all positive integers $q$ the graph $G$ is not circular $q$-degree-choosable.

If $G$ is isomorphic to $K_{4}$ then there exists no $(4 q-1, q)$-coloring of $G$. If $G$ is a tree then the required result follows from Lemma 4 and the fact that $K_{1}$ is not $l_{q^{-}}(p, q)$-choosable for all $p$. If $G$ has an odd cycle as its heart then we claim that $G$ is not $l_{q^{-}}(p, q)$-choosable for all sufficiently big $p$. By Lemma 4 it suffices to consider the case when $G$ is an odd cycle. Let $L(v)=\{0,1, \ldots, 2 q-1\}$ for all $v \in V(G)$. It is easy to see that $G$ is not $L$ - $(p, q)$-colorable. Therefore, $G$ is not $l_{q^{-}}(p, q)$-choosable for all $p \geq 2 q$, as claimed.

\section{References}

[1] O. V. Borodin. Criterion of chromaticity of a degree prescription(in russian). In Abstracts of IV All-Union Conference on Theoretical Cybernetics (Novosibirsk), pages 127-128, 1977.

[2] Paul Erdős, Arthur L. Rubin, and Herbert Taylor. Choosability in graphs. In Proceedings of the West Coast Conference on Combinatorics, Graph Theory and Computing (Humboldt State Univ., Arcata, Calif., 1979), Congress. Numer., XXVI, pages 125157, Winnipeg, Man., 1980. Utilitas Math.

[3] F. Havet, R. Kang, T. Muller, and J.-S. Sereni. Circular choosability. Submitted, available via http://hal.inria.fr/inria-00086981, 2006.

[4] B. Mohar. Choosability for the circular chromatic number. http://www .fmf .uni-lj . si/ mohar/Problems/P0201ChoosabilityCircular.html\%, 2003.

[5] Serguei Norine. On two questions about circular choosability. J. Graph Theory, 57(2):261-269, 2008.

[6] Serguei Norine, Tsai-Lien Wong, and Xuding Zhu. Circular choosability via Combinatorial Nullstellensatz. J. Graph Theory, to appear.

[7] Andre Raspaud and Xuding Zhu. List circular coloring of trees and cycles. J. Graph Theory, 55(3):249-265, 2007.

[8] Xuding Zhu. Circular choosability of graphs. J. Graph Theory, 48(3):210-218, 2005.

[9] Xuding Zhu. Recent developments in circular colouring of graphs. In M. Klazar, J. Kratochvil, J. Matousek, R. Thomas, and P. Valtr, editors, Topics in Discrete Mathematics, pages 497-550. Springer, 2006. 